\title{
Peptidoglycan Plasticity in Bacteria: Stress-Induced Peptidoglycan Editing by Noncanonical D-Amino Acids
}

\author{
Pilar Horcajo, Miguel A. de Pedro, and Felipe Cava
}

It has been generally assumed that the role of D-amino acids in bacterial physiology is rather limited. However, recent new evidence demonstrated that millimolar concentrations of noncanonical D-amino acids are synthesized and released to the environment by bacteria from diverse phyla. These D-amino acids help bacteria adapt to environmental challenges by modulating the structure and composition of the peptidoglycan (PG). This regulation, which appears to be well conserved among bacterial species, occurs principally through the incorporation of the D-amino acids into the terminus of the peptide moiety of muropeptides. These findings revived interest in studies investigating D-amino acids as an exciting and trendy topic in current microbiology, which considers them as fundamental players in different aspects of bacterial physiology. In this article, we provide an overview of the origins of research on the effects of D-amino acids in the biology of bacterial cell walls, including their recent implication as key factors for stress-associated PG remodeling.

\section{D-Amino Acids are Structural Components of Bacterial Murein}

B ACTERIA HAVE an outstanding ability to withstand many physical, chemical, and biological threats. In large part, this is due to the peptidoglycan (PG) exoskeleton, a tough multi-task matrix that envelopes the cell. Bacterial PG fortifies the cytoplasmic membrane and protects it from osmotic rupture, confers cell shape, and serves as a scaffold for anchoring other cell envelope components. ${ }^{27,58,62}$

PG consists of a basic unit made of the disaccharide $\mathrm{N}$-acetyl-glucosamine- $\mathrm{N}$-acetyl-muramic acid bound to a peptide moiety. Almost six decades ago, it was announced for the first time that two D-amino acids, D-alanine and Dglutamate, were basic components of bacterial PG. ${ }^{21,25,46}$ This discovery revealed one the most striking features of PG composition: the presence of D-forms of amino acids in the stem peptides. ${ }^{58} \mathrm{D}$-amino acids not only contribute to the architecture of the murein but also provide resistance to most known proteases. Thus, the presence of D-amino acids in PG likely constitutes a bacterial adaptation that protects a vital cellular structure. D-Ala and D-Glu are, by far, the most common D-amino acids present in the bacterial cell wall; however, the PG of some bacteria include alternative $\mathrm{D}$-amino acids in position 5, such as D-Asp in Lactococcus ${ }^{57}$ and Enterococcus, ${ }^{3}$ and D-Ser in Enterococcus gallinarum ${ }^{22}$ and in vancomycin-resistant Staphylococcus aureus. ${ }^{14,48,50}$ In some cases, such substitutions enable bacteria to tolerate bactericidal agents from the environment. ${ }^{15}$

\section{Origins of the Interest in D-Amino Acids as Modulators of Cell Wall Metabolism}

A brief overview of the historical development of research on the biological activity of D-amino acids in bacteria is quite illustrative of how science works by linking together a series of apparently disperse observations, thus leading to what looks as a blooming new field of interest.

The ability of D-amino acids to affect bacterial metabolism was first observed in 1944 when Fox and co-workers ${ }^{20}$ studied the contribution of the D-leucine residue to the antibiotic activity of gramicidin. Indeed, D-Leu, but not the L-isomer, showed a strong growth inhibitory effect on Lactobacillus arabinosus. A number of follow-up studies confirmed this finding and indicated that the D-isomers of most of the naturally occurring amino acids exhibited inhibitory or toxic activities in a number of test bacterial species. $4,23,30,34,52,56,61$ The earlier studies proposed that D-amino acids exerted their action interfering with the activity of peptidases and proteases. ${ }^{20,30,61}$ However, a more detailed analysis of the effect of D-amino acids showed that these compounds, at relatively high concentrations, were inhibitors of cell wall synthesis. ${ }^{23,34}$ Furthermore, using radioactive D-Met, Lark et al. ${ }^{33,35}$ showed that D-Met was indeed incorporated into the phenol insoluble fraction (PG) of 
bacterial cell walls, causing morphological and structural damage to the cell sacculus. Some sporadic reports in the 1960s and 1970s indicated that sensitivity to D-amino acids could be quite widespread among bacteria. $4,24,51$

The first hints on the enzymatic mechanisms of D-amino acid incorporation came when Izaki et al. ${ }^{28}$ demonstrated the ability of D-amino acids to inhibit PG biosynthesis in "in vitro" systems, and proposed that D-amino acids could reverse the final transpeptidation reaction leading to PG cross-linkage. However, it was the work of Hammes ${ }^{24}$ on PG synthesis in Aerococcus viridans var. homari (formerly Gaffkya homari) that for the first time demonstrated the ability of an L,D-peptidyl transferase, in particular an L,D-carboxypeptidase, to catalyze a $\mathrm{D}$-amino acid exchange reaction involving the C-terminal D-Ala residue of a disaccharide tetrapeptide and an exogenous D-amino acid (Fig. 1A). Later work by Tsuruoka et al. ${ }^{54}$ and Caparros et al. ${ }^{6,7}$ confirmed that the incorporation of D-amino acids in Escherichia coli was the consequence of a D-amino acid exchange reaction between C-terminal D-Ala residues in the peptide side chains of

A

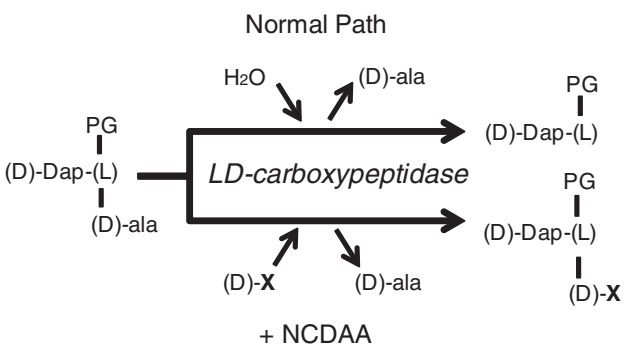

B

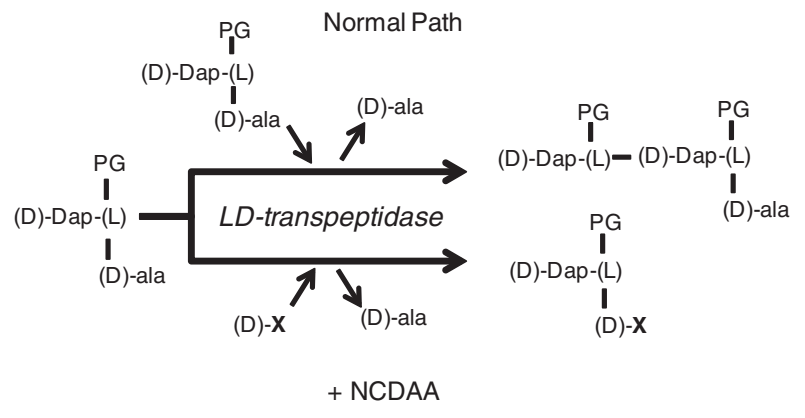

C

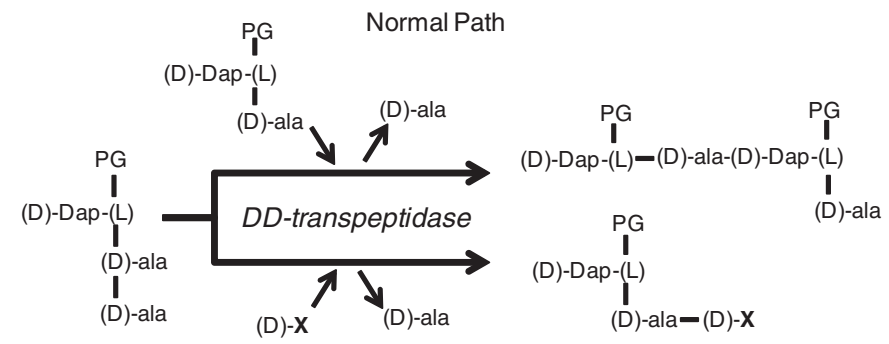

D

+ NCDAA
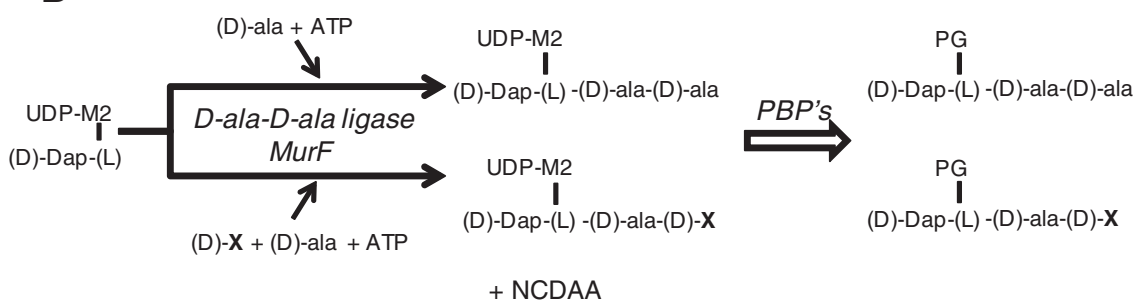

+ NCDAA

FIG. 1. Enzymatic mechanisms mediating D-amino acid incorporation into macromolecular peptidoglycan (PG). The four mechanisms with an experimental support are depicted. (A) L,D-carboxypeptidase mediated exchange reaction. ${ }^{24}$ (B) L,Dtranspeptidase-mediated exchange reaction. ${ }^{10}$ (C) D,D-transpeptidase-mediated exchange reaction. ${ }^{36}$ (D) Incorporation via modified precursors. ${ }^{10}$ PG-(D)Dap(L)-R, indicates a peptide side chain in macromolecular PG with the indicated C-terminal amino acids. UDP-M2-(D)Dap(L)-(D)-ala-R stands for UPD-muramyl pentapeptide with $\mathrm{R}$ as C-terminal amino acid. "Normal Path" corresponds to the enzymatic reaction in the absence of added noncanonical D-amino acids; "+ NCDAA" indicates the reaction in the presence of added D-amino acids. PBPs, penicillin-binding proteins. 
macromolecular PG and externally added D-amino acids. The reaction would take place in the periplasmic space mediated by a putative L,D-transpeptidase, very recently identified as the L,D-transpeptidases responsible for the synthesis of L,D-DAP-DAP cross links in both E. coli and Vibrio cholerae $^{10}$ (Fig. 1B). Current work, presented in more detail next, demonstrated the existence of distinct pathways for D-amino acid incorporation in bacteria. ${ }^{10}$ Some bacterial species are able to incorporate noncanonical D-amino acids (NCDAAs) into their sacculi through the regular biosynthetic pathway. In these instances, precursors with a residue of the NCDAA are synthesized, and then utilized as regular precursors (Fig. 1D). Furthermore, in vitro studies with purified PG synthases suggest that some D,D-transpeptidases have the potential to mediate D-amino acid exchange reactions, generating muropeptides with NCDAA at position 5 of the stem peptides ${ }^{36}$ (Fig. 1C).

The presence of NCDAA results not only in their incorporation into the cell wall, but also often in an inhibition of the rate of PG biosynthesis, possibly because of the inhibitory effects on penicillin-binding proteins (PBPs). ${ }^{7,9}$ Since this effect is not linked to a concomitant reduction in growth rate, the amount of PG per cell (per unit of cell surface, to be precise) is reduced until a new steady state is reached or the cell lyses. Interestingly, some bacteria such as E. coli are able to survive while maintaining an essentially normal morphology with severely reduced amounts of PG. ${ }^{8}$

An interesting spin off from research on the action of Damino acids was the development of a new method for the chemical labeling of bacterial sacculi "in vivo," appropriate for high-resolution visualization techniques. The method, often known as the "D-Cys labeling method," is based on the fact that as sacculi are devoid of D-Cys, an amino acid that is readily incorporated into PG by many bacteria, forcing cells to grow in its presence would result in sacculi being uniformly labeled with D-Cys. When cells are transferred to the D-Cys free medium, new PG will be devoid of D-Cys. The purification of PG and mapping the regions with and without D-Cys would then help in identifying the regions where active synthesis is taking place. D-Cys can be mapped by means of fluorescent or electro immunemicroscopy with a very high resolution. ${ }^{17}$ Application of this method led to the finding of the so-called "inert PG" regions in the bacterial sacculus, which are important for the generation of polar regions, ${ }^{16-18}$ and to a more precise definition of the topology of cell wall biosynthetic sites in different bacteria. $1,5,17$

Since the more evident effects of D-amino acids are, in most cases, deleterious to bacteria, the dominant idea was that D-amino acid incorporation into PG was the result of enzymatic side reactions with no biological meaning. This view focused research, never too intense though, on their potential as bactericidal agents, or as tools to manipulate cell shape $\mathrm{e}^{29,44,53,55,60}$ and distracted attention away from giving environmental D-amino acids a biological/ecological meaning for a long time. The discovery of their potential as signaling and regulatory molecules in $V$. cholerae, ${ }^{32}$ and next as biofilm disassembly modulators in Bacillus subtilis, ${ }^{31}$ is quickly changing this perception, and pointing to D-amino acids as a new set of flexible and likely ubiquitous regulators of growth-related phenomena.

\section{An Unexpected Turn on D-Amino Acid Research: Diverse D-Amino Acids are Released by Bacteria}

Research on D-amino acids has regained general interest by the scientific community, and particularly for microbiologists, as we reported in 2009 that unusual D-amino acids were produced by bacteria. ${ }^{32}$ This investigation revealed that diverse bacterial phyla are capable of releasing D-amino acids which are different from those that are a part of the PG. These NCDAAs are made by dedicated racemases that display multi-substrate specificity. ${ }^{32}$ In $V$. cholerae, the bacterial model of this investigation, the racemase was named BsrV, which stood for Broad Spectrum Racemase in Vibrio, and accounted for the accumulation of D-Met, D-Leu, D-Ile, and D-Val in stationary phase supernatants. ${ }^{32}$ Though NCDAAs were initially found in $V$. cholerae, their production appears to be widespread among many bacteria. In fact, most bacterial genomes appear to encode alternative amino acid racemases in addition to the enzymes required to generate D-Ala and D-Glu for PG synthesis. ${ }^{32}$ Therefore, the production of broad-spectrum racemases may be a conserved trait among diverse bacteria that have remained mis-annotated until now due to their close similarity to well-studied pyridoxal-5'-phosphate dependent-alanine racemases.

\section{Environmental Stress Triggers NCDAAs Production}

Unlike alanine and glutamate racemases, BsrV activity appears to be restricted exclusively to the stationary phase. ${ }^{32}$ This led us to investigate the putative regulators that trigger NCDAAs generation. Initially, we speculated that NCDAA production by BsrV might be induced by quorum sensing (QS) in V. cholerae. However, we immediately discarded this possibility, as $V$. cholerae N16961, the strain where NCDAA production was originally reported, ${ }^{32}$ lacks QS response due to a natural hapR frameshift mutation. Since RpoS is the transcriptional master regulator during the stationary phase, ${ }^{26}$ we decided to investigate its influence on BsrV expression. Our results showed that NCDAAs levels remained low or undetectable in the media of stationary phase rpoS mutants, indicating that RpoS influences the production of NCDAAs by BsrV. Direct regulation of the racemase by RpoS was further demonstrated using chromosomal bsrVlacZ transcription reporter fusions. ${ }^{10}$ Therefore, the fact that NCDAAs production is dependent on the alternative sigma factor RpoS, a stress-associated regulator, suggests that Damino acid synthesis and incorporation into the bacterial exoskeleton may promote bacterial resistance to diverse environmental threats.

\section{NCDAAs Govern Cell Wall Remodeling in Bacteria}

Bacterial growth and morphogenesis are intimately linked to PG synthesis and remodeling; thus, bacterial growth and survival depends on PG plasticity. ${ }^{41,42,47}$ Growth of the murein sacculus is a dynamic process that requires both synthases to make PG and incorporate it into the polymer, and hydrolytic enzymes that cleave bonds to permit expansion of the sacculus. In addition to bacterial growth, PG remodeling is crucial for many other processes such as sporulation/germination, ${ }^{49,59}$ virulence, ${ }^{3,48}$ morphological transitions, ${ }^{11,12}$ adaptation to stress conditions (i.e., stationary phase $\mathrm{e}^{43}$ ), or exposure to antibiotics. ${ }^{13}$ 
Although an understanding of the factors that govern PG remodeling during exponential growth is still limited, we have recently demonstrated that NCDAAs control changes in PG composition and architecture as cells enter the stationary phase. ${ }^{32}$ The production of NCDAAs by wild-type $V$. cholerae should have an important physiological role, as their generation represents a significant metabolic expenditure. In fact, although NCDAAs production is not essential for $V$. cholerae's viability (since still produces D-Ala and D-Glu), the $b s r V$ mutant contains twice the amount of PG found in wildtype cells in the stationary phase. Consistently, exogenous addition of physiological amounts of NCDAAs reduced the amount of PG in bsrV to wt levels. ${ }^{32}$ Therefore, NCDAAs appear to negatively regulate the amount of $\mathrm{PG}$ produced by $V$. cholerae in the stationary phase. Moreover, since the accumulation of D-amino acids coincides with the transition into the stationary phase and appears to down-regulate PG synthesis, D-amino acids may enable the coordination of metabolic slowing in cell wall and cytoplasmic compartments when resources become scarce.

Surprisingly, despite having less PG, wild-type cells are far more resistant to osmotic challenges, suggesting that the PG in wild-type cells is stronger than that in the $b s r V$ mutant. $^{32}$ Since NCDAA-containing muropeptides do not exceed a $5 \%$ of the total $V$. cholerae's stationary PG, the osmotic fragility of $b s r V$ cells is presumably more a consequence of the alterations in cell wall structure rather than in composition. In comparison to wild-type PG, the glycan chains from the $b s r V$ are shorter, and the crosslinkage is slightly higher. ${ }^{32}$ Although all these changes in PG structure and abundance appear to reduce the PG strength of the $b s r V$ mutant, it might be possible that localized modifications rather than general weakening of the entire sacculus would be sufficient to bestow osmotic sensitivity. Together, these observations suggest that D-amino acid production by BsrV as cells enter the stationary phase provides an autocrine-like signal for $V$. cholerae to remodel its PG and decrease PG synthesis in adaptation to stationary-phase conditions.

The regulation of PG by NCDAAs is not limited to $V$. cholerae. We found that B. subtilis produces different NCDAAs than $V$. cholerae (mainly D-Phe and D-Tyr) in the stationary phase, but these D-amino acids appear to influence PG synthesis and chemistry in similar ways as D-Met and D-Leu in $V$. cholerae. $^{32}$ Thus, evolutionarily distant bacteria have a common strategy to modulate PG synthesis in the stationary phase through the production and release of D-amino acids. However, contrary to $V$. cholerae, $B$. subtilis growth was inhibited when cultured in the presence of exogenous physiological concentrations of NCDAAs produced by B. subtilis in the stationary phase. $^{32}$ This suggests that $\mathrm{D}$-amino acids may be used as a mechanism that simultaneously slows down growth and PG synthesis as population density becomes saturating. The fact that, contrary to $B$. subtilis, $V$. cholerae growth is not affected by NCDAAs means that diverse sets of NCDAAs released by bacteria might act as "chemical languages" that are capable of eliciting specific behaviors/responses in subpopulations of microbes within an environmental niche.

\section{Cell Wall Editing Induced by NCDAAs}

High performance liquid chromatography (HPLC)-based analyses of muropeptides from wild-type cells demonstrated that NCDAAs were present in the PG isolated from stationary phase cells. This observation led to the hypothesis that NCDAAs might alter cell wall properties, particularly its strength, via their incorporation into the PG polymer. ${ }^{32}$ As expected from previous studies demonstrating incorporation into the PG of distinct D-amino acids at high concentrations. ${ }^{6,7,9}$ BsrV-produced D-Met is incorporated at two locations within PG subunits from the stationary phase of $V$. cholerae. ${ }^{10}$ Most of the D-Met replaces D-Ala at the fourth position of muropeptides $\left(\right.$ muro $\left.^{\mathrm{M}}\right)$; however, D-Met can also replace D-Ala at the 5 th position $\left(\right.$ muro $\left.^{\mathrm{M}}\right)$ to a lesser extent. The incorporation of D-Met into the cell wall is dependent on its production by the BsrV racemase. Furthermore, bacteria that lack a broad-spectrum racemase can, nonetheless, incorporate NCDAAs when supplied by neighbor producer organisms. ${ }^{10}$ Remarkably, all bacterial species so far studied incorporate exogenous D-amino acids into either muro4 and/or muro5 muropeptide. Although the number is still reduced, this indicates that the incorporation of NCDAAs is a widespread phenomenon. PG incorporation of NCDAAs might lead to interspecies communication, as nonproducer organisms may still be affected, beneficially or detrimentally, by NCDAAs released into the media by other bacteria in a particular niche. The possible implications of NCDAAs in the biological processes of co-inhabitants still remains to be investigated, but the enormous energy expenditure required suggests that these molecules should have a great impact in poly-microbial communities.

\section{Periplasmic L,D-Transpeptidades Incorporate NCDAAs into the Fourth Position of the Muropeptides}

The most likely pathway for the generation of muro $4^{\mathrm{M}}$ is an amino-acid exchange reaction mediated by L,D-transpeptidase enzymes (Ldt) in which the L,D-peptide bond from the L-center of meso diaminopimelic acid (DAP) is transferred to the amino group of the acceptor D-amino acid (Fig. 1B). Recent studies have revealed that Ldts catalyze the formation of DAPDAP crosslinks between muropeptides as well as the linkage between DAP and Braun's lipoprotein (Lpp) in some Gramnegative bacteria. ${ }^{38-40} \mathrm{~V}$. cholerae N16961 has two Ldt activities. ${ }^{10}$ Analysis of the mutants lacking these transpeptidases revealed that $V$. cholerae lacking vc1268 (renamed $l d t A$ ) is devoid of DAP-DAP crosslinks, whereas $V$. cholerae lacking vca0058 (renamed $l d t B$ ) lacks Lpp covalently bound to the PG. Interestingly, despite having mutually exclusive roles, both LdtA and LdtB contributed to the incorporation of NCDAAs at the fourth position within muropeptides. In contrast, neither LdtA nor LdtB appears important for the generation of muro $^{\mathrm{M}}$ peptides, which is consistent with the fact that muro $5^{\mathrm{M}}$ peptides contain a D,D-peptide bond between D-Met and the adjacent D-Ala, rather than the L,D-bond typically synthesized by Ldts. ${ }^{10}$

\section{Cytoplasmic Ligases Incorporate NCDAAs INTO PG Precursor Units}

The incorporation of NCDAA at position 5 could be explained by a parallel reasoning in which a D,D-transpeptidase could catalyze an D-amino acid exchange reaction (Fig. $1 C)$. Nevertheless, the fact that $V$. cholerae's macromolecular PG lacks pentapeptide subunits argues against this exchange reaction. ${ }^{10}$ 
As an alternative, NCDAA incorporation in muro $5^{\mathrm{M}}$ could occur through the biosynthetic pathway for the generation of cytoplasmic PG precursors (Fig. 1D). The promiscuous acceptance of alternative D-amino acids by Ddl ligases reported in some systems backs this idea. ${ }^{2}$ Indeed, muro $^{\mathrm{M}}$ synthesis was totally inhibited by D-cycloserine (a Ddl inhibitor). Furthermore, studies with an "in vitro" reaction using pure $V$. cholerae Ddl and MurF and UDP-muramyl tripeptide, D-Ala, and D-Met as substrates ${ }^{10}$ confirmed the ability of $V$. cholerae enzymes to make modified precursors, a result that strongly supports the formation of muro $5^{\mathrm{M}}$ peptides via the biosynthetic pathway in $V$. cholerae.

\section{Different Processes Control Formation of D-MET Muropeptides}

As mentioned earlier, all bacteria grown in media supplemented with exogenous NCDAAs showed incorporation into their PG, indicating that the mechanism/s might be well conserved among bacteria. Except $V$. cholerae and Caulobacter crescentus, which incorporate NCDAAs into both tetrapeptides and pentapeptides, all the other species tested displayed only one class of NCDAA-containing muropeptides. ${ }^{10}$

As for $V$. cholerae, the muro $4^{\mathrm{M}}$ is also generated in other bacteria by Ldt orthologs, as HPLC analysis of the PG isolated from Ldt mutants in media supplemented with exogenous D-Met exhibited reduced exchange of D-Ala by D-Met. Accordingly, organisms devoid of Ldt homologues have no D-Met incorporated into tetrapeptides even at high concentrations (Cava et al. ${ }^{10}$ and unpublished data).

Similarly, we investigated whether muro $^{\mathrm{M}}$ formation through Ddl and MurF is also conserved among bacterial species. In fact, muro $5^{\mathrm{M}}$ accumulation resulted clearly inhibited by D-cycloserine in some organisms such as C. crescentus or S. aureus (Cava et al. ${ }^{10}$ and unpublished results). Surprisingly, muro $^{\mathrm{M}}$ generation in $B$. subtilis was insensitive to D-cycloserine, suggesting a different enzymatic pathway, likely dependent on penicillin-binding D,D-peptidyl transferases. ${ }^{10}$ Therefore, unlike muro ${ }^{\mathrm{M}}$ peptides, it appears that muro5 $^{\mathrm{M}}$ peptides can arise via multiple processes. In this regard, it has been recently reported that E. coli transpeptidase PBP1A can incorporate D-amino acids into pentapeptides in vitro by using PG fragments. ${ }^{36}$ This suggests that for some organisms such as E. coli, the modification of pentapeptides by NCDAAs might have been excluded from mature PG due to the action of D,D-carboxypeptidases, whereas in $V$. cholerae, these enzymes might discriminate between canonical and noncanonical pentapeptides. Whatever the case is, it still remains to be seen whether muro $5^{\mathrm{x}}$ formation in vivo is predominantly performed by periplasmic D,D-transpeptidases or by cytosolic biosynthetic ligases.

\section{Incorporation of NCDAA Triggers Cell Wall Regulation}

Remarkably, results obtained with Ldt mutants showed that NCDAA incorporation is the major mechanism used by $V$. cholerae that controls PG amount and strength (osmotic sensitivity) in the stationary phase. ${ }^{10}$ However, it is unlikely that all the differences in PG composition, structure, and amount observed between $V$. cholerae wild-type and $b s r V$ mutant strains can be solely attributed to the incorporation of D-amino acids into PG. Supporting this idea, D- but not L-Met blocks the binding of a fluorescent derivative of
Penicillin G to several $V$. cholerae PBPs. ${ }^{32}$ This result suggests that free amino acids accumulated in the periplasm might compete with muropeptide moieties for PBP active sites, thereby serving as regulators of the enzymes that synthesize and modify the PG polymer.

In agreement with this idea, other investigators have observed in the past a synergistic effect of exogenous D-amino acids in combination with $\beta$-lactam antibiotics ${ }^{34,56}$ that strongly supports NCDAAs as direct regulators of PBP activities.

\section{Summary and Concluding Remarks}

For a long time, it was appreciated that amino acids are presented in nature in two different sets of enantiomeric isomers. However, contrary to the interest attracted by the L-forms, the biological function of D-amino acids remained mysterious and mainly reduced to a few exceptions related to their presence in bacterial PG and in some nonribosomal peptides.

Starting in the mid twentieth century, numerous researchers reported notable observations about the deleterious effects of exogenous D-amino acids in bacteria. However, similar to other bacterial secondary metabolites whose biological function was believed to be merely antimicrobial in the past, recent investigations have unveiled remarkable regulatory activities for D-amino acids at physiological (environmental) doses.

Released D-amino acids permit bacterial cell wall adaptation to adverse environments. BsrV expression is fully dependent on RpoS, the sigma factor that helps many bacterial pathogens respond to different environmental stress conditions, such as those associated with entry into the stationary phase (where D-amino acids were first detected) and those that contribute to the expression of virulenceassociated genes. ${ }^{19,37,45} \mathrm{BsrV}$ under the control of RpoS opens a wide spectrum of challenging situations (such as an infection, temperature, and salinity fluctuations, or the constant battle that prevails in a niche) where NCDAAs may help bacteria to adapt to adverse conditions. When facing environmental threats, the normal growth of bacteria can be altered, often leading to complete arrest. In this context, bacteria need to synchronize the activities at both sides of the cytoplasmic membrane and this is exactly when D-amino acids display a fundamental role in overcoming the challenge of controlling PG metabolism.

In addition, an important aspect of bacterial stress response, often overlooked, is the generation of morphological modifications and the differentiation of new structures derived or connected with the cell wall. Indeed, most bacteria alter shape when growth conditions change and, even more drastically, on interaction with other organisms in the course of symbiotic or parasitic processes. Any modification in cell shape is intimately connected with cell wall metabolism and, therefore, stress-dependent morphological modifications require specific regulatory mechanisms still poorly understood. Although a stationary phase-dependent morphological reduction in $V$. cholerae appears not to be dependent on BsrV, it remains to be seen whether the released D-amino acids have the potential to mediate other stress-dependent morphological changes in bacteria.

PG regulation by NCDAAs occurs mainly through the generation of modified muropeptides, which, acting as 
nonpreferred substrates, might slow down the activity of the PBPs and, thus, PG biosynthesis rate. Controlling the concentration of NCDAAs in the periplasm could constitute a reversible mechanism for inhibiting PBPs, thereby enabling cells to rapidly transition between states of active and inactive PG metabolism under changing environmental conditions. Furthermore, since D-amino acids are released to the media, this regulation permits the coordinated response of the entire population rather than an individual regulation to environmental changes.

The fact that most bacteria present a murein cell wall implies that this regulatory mechanism should be quite general. Supporting this, many diverse bacteria encode broad-spectrum racemase homologues in their genomes and, thus, are virtually capable of self-controlling their PG through D-amino acid production. However, the mechanism used by bacterial species lacking broad-spectrum racemases that controls their PG under stress conditions is not clear.

Since a great variety of diverse microbes co-exist in an environmental niche, some bacteria might have evolved as nonproducers, passing the responsibility of D-amino acids production to other members of the poly-microbial community. This is feasible only if nonproducer strains can gain advantage of the paracrine production of D-amino acids by nearby bacteria. In this regard, the mechanism of D-amino acids incorporation into the murein sacculus is very well conserved in bacteria, as it is catalyzed by enzymes that participate in PG biosynthesis. In fact, both L,D-transpeptidases and cytoplasmic PG ligases have been reported as having multi-substrate specificity and are, thus, capable of accepting alternative D-amino acids, such as those released by diverse types of bacteria, to remodel PG composition and structure. Therefore, PG modulation might be a common behavior in poly-microbial communities through paracrine signaling. However, it remains to be seen what mechanism/s might control PG slowdown in monocultures of bacteria lacking broad-spectrum racemases.

Finally, oncoming investigation will also shed light on the leading role of PG-hydrolytic enzymes (D,D-carboxypeptidases and L,D-carboxypeptidases) as additional checkpoints used by bacteria to control the activity of NCDAAmuropeptides.

The discovery of further roles of D-amino acids as community-signaling effectors would certainly be a major breakthrough in microbial ecology and physiology. However, the case of NCDAA is probably only one of the numerous mechanisms awaiting to be discovered and involved in PG cross-regulation within complex microbial communities. Therefore, the investigation of cell wall biochemistry under natural conditions, and in a large number of organisms, is imperative for a realistic understanding of cell wall biology, in particular with regard to its role in environmental adaptation and signaling.

\section{Acknowledgments}

Substantial parts of this work were performed by F.C. at Matthew K. Waldor's Laboratory in Harvard Medical School and supported by Howard Hughes Medical Institute (HHMI); Ministry of Education and Science, Spain (MEC) BFU2006-04574; Fundación Ramón Areces (M.A.P.); MICINN Ramon y Cajal Program-UAM (F.C.); and UAM (P.H.).

\section{Disclosure Statement}

The authors declare no conflict of interest.

\section{References}

1. Aaron, M., G. Charbon, H. Lam, H. Schwarz, W. Vollmer, and C. Jacobs-Wagner. 2007. The tubulin homologue FtsZ contributes to cell elongation by guiding cell wall precursor synthesis in Caulobacter crescentus. Mol. Microbiol. 64: 938-952.

2. Barreteau, H., A. Kovac, A. Boniface, M. Sova, S. Gobec, and D. Blanot. 2008. Cytoplasmic steps of peptidoglycan biosynthesis. FEMS Microbiol. Rev. 32:168-207.

3. Bellais, S., M. Arthur, L. Dubost, J.E. Hugonnet, L. Gutmann, J. van Heijenoort, R. Legrand, J.P. Brouard, L. Rice, and J.L. Mainardi. 2006. Aslfm, the D-aspartate ligase responsible for the addition of D-aspartic acid onto the peptidoglycan precursor of Enterococcus faecium. J. Biol. Chem. 281:11586-11594.

4. Bopp, M. 1965. [Inhibition of Agrobacterium tumefaciens by D-amino acids]. Z Naturforsch B 20:899-905.

5. Brown, P.J.B., M.A. de Pedro, D.T. Kysela, C. Van der Henst, J. Kim, X. De Bolle, C. Fuqua, and Y.V. Brun. 2012. Polar growth in the Alphaproteobacterial Order Rhizobiales. Proc. Natl. Acad. Sci. U. S. A. 109:1697-1701.

6. Caparros, M., V. Aran, and M.A. de Pedro. 1992. Incorporation of S-[3H]methyl-D-cysteine into the peptidoglycan of ether-treated cells of Escherichia coli. FEMS Microbiol. Lett. 72:139-146.

7. Caparros, M., A.G. Pisabarro, and M.A. de Pedro. 1992. Effect of D-amino acids on structure and synthesis of peptidoglycan in Escherichia coli. J. Bacteriol. 174:5549-5559.

8. Caparros, M., J.C. Quintela, and M.A. de Pedro. 1994. Variability of peptidoglycan surface density in Escherichia coli. FEMS Microbiol. Lett. 121:71-76.

9. Caparros, M., J.L. Torrecuadrada, and M.A. de Pedro. 1991. Effect of D-amino acids on Escherichia coli strains with impaired penicillin-binding proteins. Res. Microbiol. 142: 345-350.

10. Cava, F., M.A. de Pedro, H. Lam, B.M. Davis, and M.K. Waldor. 2011. Distinct pathways for modification of the bacterial cell wall by non-canonical D-amino acids. EMBO J. 30:3442-3453.

11. Chaput, C., A. Labigne, and I.G. Boneca. 2007. Characterization of Helicobacter pylori lytic transglycosylases Slt and MltD. J. Bacteriol. 189:422-429.

12. Costa, K., G. Bacher, G. Allmaier, M.G. DominguezBello, L. Engstrand, P. Falk, M.A. de Pedro, and F. Garciadel Portillo. 1999. The morphological transition of Helicobacter pylori cells from spiral to coccoid is preceded by a substantial modification of the cell wall. J. Bacteriol. 181: 3710-3715.

13. Davis, K.M., and J.N. Weiser. 2011. Modifications to the peptidoglycan backbone help bacteria to establish infection. Infect. Immun. 79:562-570.

14. De Jonge, B.L., D. Gage, and N. Xu. 2002. The carboxyl terminus of peptidoglycan stem peptides is a determinant for methicillin resistance in Staphylococcus aureus. Antimicrob. Agents Chemother. 46:3151-3155.

15. de Lencastre, H., D. Oliveira, and A. Tomasz. 2007. Antibiotic resistant Staphylococcus aureus: a paradigm of adaptive power. Curr. Opin. Microbiol. 10:428-435.

16. de Pedro, M.A., W.D. Donachie, J.V. Holtje, and H. Schwarz. 2001. Constitutive septal murein synthesis in 
Escherichia coli with impaired activity of the morphogenetic proteins RodA and penicillin-binding protein 2. J. Bacteriol. 183:4115-4126.

17. de Pedro, M.A., J.C. Quintela, J.V. Holtje, and H. Schwarz. 1997. Murein segregation in Escherichia coli. J. Bacteriol. 179:2823-2834.

18. de Pedro, M.A., K.D. Young, J.V. Holtje, and H. Schwarz. 2003. Branching of Escherichia coli cells arises from multiple sites of inert peptidoglycan. J. Bacteriol. 185:1147-1152.

19. Fang, F.C., S.J. Libby, N.A. Buchmeier, P.C. Loewen, J. Switala, J. Harwood, and D.G. Guiney. 1992. The alternative sigma factor katF (rpoS) regulates Salmonella virulence. Proc. Natl. Acad. Sci. U. S. A. 89:11978-11982.

20. Fox, S.W., M. Fling, and N. Bollenback. 1944. Inhibition of bacterial growth by d-leucine. J. Biol. Chem. 155: 465-468.

21. Ghuysen, J.M. 1961. [Data on the structure of disaccharidepeptide complexes liberated from the wall of Micrococcus lysodeikticus by the action of beta(1-4)N-acetylhexosaminidases]. Biochim Biophys Acta 47:561-568.

22. Grohs, P., L. Gutmann, R. Legrand, B. Schoot, and J.L. Mainardi. 2000. Vancomycin resistance is associated with serine-containing peptidoglycan in Enterococcus gallinarum. J. Bacteriol. 182:6228-6232.

23. Grula, E.A. 1960. Cell division in a species of Erwinia. I. Inhibition of division by D-amino acids. J. Bacteriol. 80: 375-385.

24. Hammes, W.P. 1978. The LD-carboxypeptidase activity in Gaffkya homari. The target of the action of D-amino acids or glycine on the formation of wall-bound peptidoglycan. Eur. J. Biochem. 91:501-507.

25. Hancock, R. 1960. The amino acid composition of the protein and cell wall of Staphylococcus aureus. Biochim. Biophys. Acta. 37:42-46.

26. Hengge-Aronis, R. 2002. Recent insights into the general stress response regulatory network in Escherichia coli. J. Mol. Microbiol. Biotechnol. 4:341-346.

27. Holtje, J.V. 1998. Growth of the stress-bearing and shapemaintaining murein sacculus of Escherichia coli. Microbiol. Mol. Biol. Rev. 62:181-203.

28. Izaki, K., M. Matsuhashi, and J.L. Strominger. 1968. Biosynthesis of the peptidoglycan of bacterial cell walls. 8 . Peptidoglycan transpeptidase and D-alanine carboxypeptidase: penicillin-sensitive enzymatic reaction in strains of Escherichia coli. J. Biol. Chem. 243:3180-3192.

29. Janssen, P.H., L.E. Parker, and H.W. Morgan. 1991. Filament formation in Thermus species in the presence of some D-amino acids or glycine. Antonie Van Leeuwenhoek 59:147-154.

30. Kobayashi, Y., M. Fling, and S.W. Fox. 1948. Antipodal specificity in the inhibition of growth of Escherichia coli by amino acids. J. Biol. Chem. 174:391-398.

31. Kolodkin-Gal, I., D. Romero, S. Cao, J. Clardy, R. Kolter, and R. Losick. 2010. D-amino acids trigger biofilm disassembly. Science 328:627-629.

32. Lam, H., D.C. Oh, F. Cava, C.N. Takacs, J. Clardy, M.A. de Pedro, and M.K. Waldor. 2009. D-amino acids govern stationary phase cell wall remodeling in bacteria. Science 325:1552-1555.

33. Lark, C., D. Bradley, and K.G. Lark. 1963. Further studies on the incorporation of D-methionine into the bacterial cell wall; its incorporation into the R-layer and the structural consequences. Biochim. Biophys. Acta. 78:278-288.
34. Lark, C., and K.G. Lark. 1959. The effects of D-amino acids on Alcaligenes fecalis. Can. J. Microbiol. 5:369-379.

35. Lark, C., and K.G. Lark. 1961. Studies on the mechanism by which D-amino acids block cell wall synthesis. Biochim. Biophys. Acta. 49:308-322.

36. Lupoli, T.J., H. Tsukamoto, E.H. Doud, T.S. Wang, S. Walker, and D. Kahne. 2011. Transpeptidase-mediated incorporation of D-amino acids into bacterial peptidoglycan. J. Am. Chem. Soc. 133:10748-10751.

37. Ma, L., J. Chen, R. Liu, X.H. Zhang, and Y.A. Jiang. 2009. Mutation of rpoS gene decreased resistance to environmental stresses, synthesis of extracellular products and virulence of Vibrio anguillarum. FEMS Microbiol. Ecol. 70:130-136.

38. Magnet, S., A. Arbeloa, J.L. Mainardi, J.E. Hugonnet, M. Fourgeaud, L. Dubost, A. Marie, V. Delfosse, C. Mayer, L.B. Rice, and M. Arthur. 2007. Specificity of L,Dtranspeptidases from gram-positive bacteria producing different peptidoglycan chemotypes. J. Biol. Chem. 282:1315113159.

39. Magnet, S., S. Bellais, L. Dubost, M. Fourgeaud, J.L. Mainardi, S. Petit-Frere, A. Marie, D. Mengin-Lecreulx, M. Arthur, and L. Gutmann. 2007. Identification of the L,Dtranspeptidases responsible for attachment of the Braun lipoprotein to Escherichia coli peptidoglycan. J. Bacteriol. 189:3927-3931.

40. Magnet, S., L. Dubost, A. Marie, M. Arthur, and L. Gutmann. 2008. Identification of the L,D-transpeptidases for peptidoglycan cross-linking in Escherichia coli. J. Bacteriol. 190:4782-4785.

41. Mengin-Lecreulx, D., and B. Lemaitre. 2005. Structure and metabolism of peptidoglycan and molecular requirements allowing its detection by the Drosophila innate immune system. J. Endotoxin Res. 11:105-111.

42. Nanninga, N. 1998. Morphogenesis of Escherichia coli. Microbiol. Mol. Biol. Rev. 62:110-129.

43. Nystrom, T. 2004. Stationary-phase physiology. Annu. Rev. Microbiol. 58:161-181.

44. Ottolenghi, A.C., M. Caparros, and M.A. de Pedro. 1993. Peptidoglycan tripeptide content and cross-linking are altered in Enterobacter cloacae induced to produce AmpC beta-lactamase by glycine and D-amino acids. J. Bacteriol. 175:1537-1542.

45. Ouyang, Z., R.K. Deka, and M.V. Norgard. 2011. BosR (BB0647) controls the RpoN-RpoS regulatory pathway and virulence expression in Borrelia burgdorferi by a novel DNA-binding mechanism. PLoS Pathog. 7:e1001272.

46. Park, J.T., and J.L. Strominger. 1957. Mode of action of penicillin. Science 125:99-101.

47. Park, J.T., and T. Uehara. 2008. How bacteria consume their own exoskeletons (turnover and recycling of cell wall peptidoglycan). Microbiol. Mol. Biol. Rev. 72:211-227, table of contents.

48. Reynolds, P.E., and P. Courvalin. 2005. Vancomycin resistance in enterococci due to synthesis of precursors terminating in D-alanyl-D-serine. Antimicrob. Agents Chemother. 49:21-25.

49. Setlow, P. 2003. Spore germination. Curr. Opin. Microbiol. 6:550-556.

50. Sieradzki, K., and A. Tomasz. 1996. A highly vancomycinresistant laboratory mutant of Staphylococcus aureus. FEMS Microbiol. Lett. 142:161-166.

51. Strijdom, B.W., and O.N. Allen. 1966. Medium supplementation with $\mathrm{L}$ - and $\mathrm{D}$-amino acids relative to growth and efficiency of Rhizobium meliloti. Can. J. Microbiol. 12:275-283. 
52. Teeri, A.E. 1954. Effect of D-amino acids on growth of Lactobacilli. J. Bacteriol. 67:686-688.

53. Tsuruoka, T., A. Tamura, A. Miyata, T. Takei, S. Inouye, and M. Matsuhashi. 1985. Second lytic target of beta-lactam compounds that have a terminal D-amino acid residue. Eur. J. Biochem. 151:209-216.

54. Tsuruoka, T., A. Tamura, A. Miyata, T. Takei, K. Iwamatsu, S. Inouye, and M. Matsuhashi. 1984. Penicillin-insensitive incorporation of D-amino acids into cell wall peptidoglycan influences the amount of bound lipoprotein in Escherichia coli. J. Bacteriol. 160:889-894.

55. Tuomanen, E., and A. Tomasz. 1984. Protection by D-amino acids against growth inhibition and lysis caused by betalactam antibiotics. Antimicrob. Agents Chemother. 26: $414-416$.

56. Tuttle, A.L., and H. Gest. 1960. Induction of morphological aberrations in Rhodospirillum rubrum by D-amino acids. J. Bacteriol. 79:213-216.

57. Veiga, P., S. Piquet, A. Maisons, S. Furlan, P. Courtin, M.P. Chapot-Chartier, and S. Kulakauskas. 2006. Identification of an essential gene responsible for D-Asp incorporation in the Lactococcus lactis peptidoglycan crossbridge. Mol. Microbiol. 62:1713-1724.
58. Vollmer, W., D. Blanot, and M.A. de Pedro. 2008. Peptidoglycan structure and architecture. FEMS Microbiol. Rev. 32:149-167.

59. Vollmer, W., B. Joris, P. Charlier, and S. Foster. 2008. Bacterial peptidoglycan (murein) hydrolases. FEMS Microbiol. Rev. 32:259-286.

60. Wecke, J., T. Kersten, and P. Giesbrecht. 1992. The modulation of the bacteriolytic effect of beta-lactam antibiotics by non-antibiotics. APMIS Suppl 30:32-39.

61. Yaw, K.E., and J.C. Kakavas. 1952. Studies on the effects of D-amino acids on Brucella abortus. J. Bacteriol. 63:263-268.

62. Young, K.D. 2006. The selective value of bacterial shape. Microbiol. Mol. Biol. Rev. 70:660-703.

Address correspondence to: Felipe Cava, Ph.D. Centro de Biología Molecular "Severo Ochoa" Universidad Autónoma de Madrid-Consejo Superior de Investigaciones Científicas Madrid 28049

Spain

E-mail: fcava@cbm.uam.es 